\title{
Special issue on 3D acquisition technology for cultural heritage
}

\section{Other Journal Item}

\section{Author(s):}

Van Gool, Luc; Sablatnig, Robert

Publication date:

2006-12

Permanent link:

https://doi.org/10.3929/ethz-b-000035742

\section{Rights / license:}

In Copyright - Non-Commercial Use Permitted

\section{Originally published in:}

Machine Vision and Applications 17(6), https://doi.org/10.1007/s00138-006-0042-2 


\title{
Special issue on 3D acquisition technology for cultural heritage
}

\author{
Luc Van Gool • Robert Sablatnig
}

Published online: 3 October 2006

(C) Springer-Verlag 2006

Computer Graphics has been used to aid in the study and presentation of Cultural Heritage for a long time now. Increasingly, also Computer Vision starts to play its role, as a matter of fact often in combination with graphics. This has lead the International Association for Pattern Recognition (IAPR) to create its Technical Committee 19 'Computer Vision for Cultural Heritage Applications' (http://iapr-tc19.prip.tuwien.ac.at/), co-chaired by the editors of this special issue. About the same time, the creation of the European Network of Excellence EPOCH (Excellence in the Processing of Open Cultural Heritage - http://www.epoch-net.org) has given this specific domain of research an additional impetus in Europe, but also beyond.

It therefore seemed timely to devote one or more special issues of Machine Vision and Applications to Cultural Heritage $(\mathrm{CH})$. In this first issue, the reader finds papers describing several state-of-the-art 3D acquisition techniques, designed or at least selected for $\mathrm{CH}$ applications.

The authors hold IAPR TC 19 co-chairs.

L. Van Gool $(\bowtie)$

Department of Information Technology and Electrical Engineering, Computer Vision Laboratory, ETH Zurich,

Sternwartstrasse 7, 8092 Zurich, Switzerland

e-mail:vangool@vision.ee.ethz.ch

L. Van Gool

ESAT-PSI-Visics, Katholieke Universiteit Leuven,

Kasteelpark Arenberg 10, 3001 Leuven, Belgium

R. Sablatnig

Institute of Computer Aided Automation,

Pattern Recognition and Image Processing Group,

Vienna University of Technology, Favoritenstrasse 9/183-2,

1040 Vienna, Austria
Our $\mathrm{CH}$ is intersected by many interests, including those of arts, sciences, resource planning and development and monument preservation, and is exposed to the adverse effects of more recent building activities, tourism, ageing, etc. Since the intensity of these risks is continually increasing, preservation and research are especially urgent. New technologies, and especially 3D scanning and virtual reconstruction, are already proving useful in helping to preserve and investigate our $\mathrm{CH}$.

In recent years, a number of large-scale projects dedicated to archaeological site modelling or museum collection digitization have emerged as an important driving force for major contributions in the field. The $\mathrm{CH}$ area is at a point where it can benefit greatly from the application of computer vision methods, and in turn provides a large number of new, challenging, interesting conceptual problems and data for computer vision.

As to the content of this issue, $\mathrm{CH}$ is an ideal testing ground for 3D acquisition technology. Not only are the objects and sites to be reconstructed often of a stunning beauty, but $\mathrm{CH}$ is also incredibly demanding. It presents these 3D systems with a combination of requirements that are hard to meet. Surfaces can have a wide range of characteristics, from shiny and transparent-like glass to extremely fine-grained-like fur or textile. Shapes may be highly complicated, with narrow protrusions and cavities, or extremely simple-like purely planar and untextured, which may create problems also. Pieces can be priceless and sites may be remote, so the system should be easy to transport to the objects of interest. Given the fragility of many of the objects, it must not make contact. As to the system itself, it should be rugged enough to work in the open, under adverse conditions. Moreover, the local and global precision has to be high as the models are an important record of the object meant 
for posterity and not just a tool. In the same vein, apart from the shape, also the surface colors and texture need to be captured in the greatest detail. The requirements that the devices should be handled by non-engineers and that they should be cheap to be affordable for these applications add to this, and 'mission impossible' may cross one's mind.

This being said, important contributions have been made lately, maybe not delivering the total package yet, but at least offering excellent tools that bring us further along several of these requirement axes.

The issue starts off with the description of a 3D acquisition campaign by Guidi et al., who combined existing laser-based scanning apparatus to produce a model of the Plastico di Roma Antica. The problem here was to achieve high precision for a very large object. Landon and Seales show how off-the-shelf components could be assembled to build a portable, triangulation-based scanner that yields both detailed shape and texture.
They demonstrate its use in the on-site digitization of Puerto Rican petroglyphic iconography. Brusco et al. push the boundaries of combined shape and texture capture further, by following multi-spectral reflectance measurements. This allows for a faithful visualization under a wide range of virtual lighting conditions. Blais and Beraldin developed a system that eases the process of on-site calibration and even compensates for relative motions of the scanner and the object. They also suggest optimal configurations of the setup in order to ensure excellent results under on-site scanning conditions. Finally, the last paper presents a passive technique, i.e. a technique that doesn't use special illumination like lasers or structured light. Vergauwen and Van Gool present a passive acquisition technique, that allows users to upload images taken with a normal video or still camera to a website, which subsequently extracts $3 \mathrm{D}$ data fully automatically and makes them available to the user via a simple downloading procedure. 